\title{
C-15
}

\section{METODOLOGÍA PARA LA OPTIMIZACIÓN CONJUNTA DE LA PROGRAMACIÓN DEL RIEGO Y ESTACIONES DE BOMBEO EN BOMBEOS DIRECTOS}

\author{
Faci, $E^{1}$, Seral, $P^{2}$, Zorrilla $F^{2}$, Garcia, $A^{2}$, Aliod $R^{2}$, García Asín, $S^{2}$ \\ ${ }^{1}$ Ing. Químico, estudiante doctorado Ingeniería Mecánica. Escuela de Ingeniería y \\ Arquitectura. Zaragoza efaci@unizar.es \\ ${ }^{2}$ Área de Mecánica de Fluidos. Grupo I+D+I GESTAR Escuela Politécnica Superior \\ Huesca.,raliod@unizar.es,susana.garcia@unizar.es
}

\section{Resumen}

Se describe una nueva metodología para reducción de los costes energéticos de operación del riego en redes de riego con bombeo directo, contemplando la optimización conjunta de la organización de los riegos y la modulación de las presiones de la estación de bombeo a lo largo de diferentes periodos tarifarios. Se describe el funcionamiento del método de optimización investigado, que es una extensión y mejora del algoritmo inicial existente desarrollado en (Faci E., 2014) destacando las diferencias y mejoras. Por último, se muestran los resultados en el caso de la red de la CR Callén (Huesca), y se comparan con los obtenidos en un estudio para la optimización energética utilizando el algoritmo inicial.

\begin{abstract}
A new methodology to reduce energy cost in irrigation networks is developed in this paper. Its goal is to optimize simultaneously both pumping station and hydrant operation by setting different discharge pressure on the pumping station and distributing adequately hydrant irrigation during different tariff periods. This methodology is an extension an existing one described in Faci E., (2014) which is only capable of optimize hydrant operation. Advantages and differences between them are described and they are compared in the case study of CR Callén network (Huesca) which shows the new methodology is capable to save an additional $10 \%$ of the annual electricity costs.
\end{abstract}

\section{1.- Introducción, Objetivos}

El objeto de la presente comunicación es describir una nueva metodología para reducción de los costes energéticos de operación del riego en redes de riego con bombeo directo, contemplando la optimización conjunta de la organización de los riegos y la modulación de las presiones de la estación de bombeo a lo largo de diferentes periodos tarifarios.

Se describe el funcionamiento del método de optimización investigado, que es una extensión y mejora del algoritmo inicial existente desarrollado en (Faci, 2014) y con ejemplos de explotación en (Aliod et al. 2015) y (García et al., 2015) en que se optimizaban las peticiones de riego, pero la modulación de presiones de la estación de bombeo era un dato impuesto, destacando las diferencias y mejoras. 
Por último, se muestran los resultados en el caso de la red de la CR Callén (Huesca), y se comparan con los obtenidos en un estudio para la optimización energética utilizando el algoritmo inicial.

\section{2.- Materiales y Métodos}

Se ha construido una extensión del sistema de optimización de programaciones de riego en redes con bombeo directo desarrollado (Faci, 2014)

En el algoritmo original permite optimizar únicamente el momento de apertura y cierre de hidrantes, de modo que la curva de consigna de la estación de bombeo tiene que ser conocida a priori.

Se observó que en algunos estudios era necesario calcular la altura de bombeo de las estaciones de bombeo. Esto requería un proceso iterativo manual en el que se asignan diferentes consignas y elegir aquella que diera mejor resultado.

Por ello, se ha desarrollado una extensión al algoritmo, que es capaz de suplir esta carencia, incorporando como variable de decisión los parámetros de la curva de consigna de las bombas además de los momentos de apertura y cierre que ya incluía el original.

El modelo que representa la bondad de una programación de riego se compone de una suma de funciones validadoras que ponderan distintos aspectos hidráulicos y energéticos del sistema tales como: presión en los hidrantes, velocidad en las tuberías, coste energético, entre otros.

La jornada de riego se discretiza en intervalos de tiempo iguales. En cada uno de ellos, se calcula el estado hidráulico del sistema y se evalúan las funciones validadoras, de modo que la bondad o función objetivo de una jornada de riego se define según la Eq 1.

$$
\text { función objetivo }=\Delta t \cdot \sum_{\mathrm{i}=0}^{\mathrm{N} n \mathrm{n}} \sum_{\mathrm{j}=0}^{\mathrm{NVal}} \mathrm{f}_{\mathrm{j}}\left(\mathrm{t}_{\mathrm{i}}\right)
$$

$\Delta \mathrm{t}$ : duración del intervalo de discretizacion de la jornada de riego

NInt : numero total de intervalos en los que se ha discretizado la jornada de riego

$\mathrm{NVal}$ : número de funciones validadoras.

$\mathrm{f}_{\mathrm{j}}\left(\mathrm{t}_{\mathrm{i}}\right)$ : valor de la función validadora $\mathrm{i}$ - esima en el intervalo $\mathrm{j}$ - esimo

Eq 1. Definición de la función objetivo

Para cada intervalo temporal i-ésimo se evalúan cada una de las funciones validadoras j-ésimas en el instante $t_{i}$. Este valor es el que se pretende minimizar. Las variables independientes del problema son:

- La hora de apertura de cada hidrante

- El valor de los coeficientes: $\operatorname{coef}_{1}, \ldots, \operatorname{coef}_{n}$ que definen la curva de consigna $\mathrm{H}=\mathrm{f}\left(\mathrm{Q}, \operatorname{coef}_{1}, \ldots, \operatorname{coef}_{\mathrm{n}}\right)$ de la estación de bombeo. El algoritmo soporta cualquier tipo de curva cuya definición sea explícita. 
El algoritmo para la optimización es de tipo metaheurístico basado en el Ant Colony descrito por (Dorigo, 1992). con optimización local mediante Hill-Climbing. Se ha implementado en C\# (.NET 4.6) y permite el uso de diferentes librerías de cálculo hidráulico como Epanet (Rossman L.) y Netcal (Estrada et al., 2009) desarrollada en el grupo GESTAR.

\section{3.- Caso de estudio}

Se realizó un estudio, año 2014, efectuado para el rediseño de la red de la CRR Callén (Huesca), que consta de 57 hidrantes que riegan una superficie de 698 ha, utilizando el algoritmo original. En tal estudio, las presiones de la estación de bombeo, variables en el tiempo, eran impuestas como condiciones forzadas de explotación, previamente ajustadas mediante tanteos.

El objetivo del estudio era proponer dos niveles de presión en la estación de bombeo, dependiendo del periodo tarifario de modo que se consiguiera un ahorro energético, teniendo en cuenta también el término de potencia, manteniendo una buena calidad de servicio (presión en hidrante suficiente).

En este nuevo estudio, se considera la siguiente curva de consigna constante para cada nivel de presión, de modo que durante P6 la altura de bombeo será $\operatorname{coef}_{1}, \mathrm{y}$ durante P5 hasta P2 será coef $_{2}$ tal y como muestran Eq. 2 y Fig 1:

$$
\mathrm{H}(\mathrm{t})=\left\{\begin{array}{ccc}
\text { coef }_{1} & \text { si } & \mathrm{t} \subset \mathrm{P} 6 \\
0 & \text { si } & \mathrm{t} \subset \mathrm{P} 1 \\
\text { coef }_{2} & \text { si } & \mathrm{t} \subset \mathrm{P} 2-\mathrm{P} 5
\end{array}\right.
$$

Eq. 2 Curva de consigna de la EB

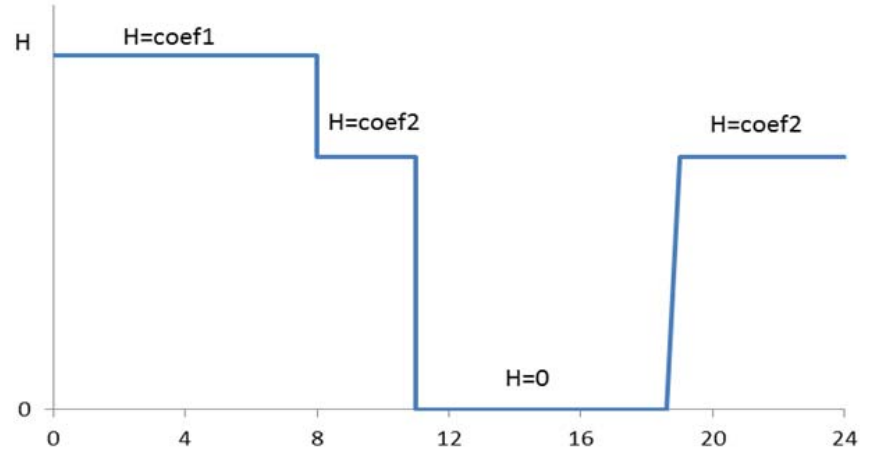

Figura 1. Curva de consigna tipo de la EB en el mes de Julio.

Se optimizará de manera conjunta un día tipo de los meses que tienen relevancia en el riego: mayo, primera quincena de junio, segunda quincena de junio, julio, agosto y septiembre. Se ponderan teniendo en cuenta el número de días de cada mes, de modo que se obtiene el coste anual equivalente.

El caso de estudio pretende optimizar simultáneamente:

1. Apertura y cierre anual de los hidrantes

2. Punto de funcionamiento de la estación de bombeo en alta presión (P3-P6) y el de baja presión (P2).

3. Potencia contratada.

Por ello, se construye la función objetivo de la Eq. 3. 


$$
f \text { obj. }=f_{\text {energia }}+f_{\text {servicio }}+f_{\text {potencia }}
$$

\section{Eq 3. Función objetivo.}

La función validadora de coste energético evalúa la energía consumida y evalúa su coste dependiendo del periodo tarifario en el que sea consumida tal y como se muestra en la Eq.4:

$$
\begin{aligned}
& \mathrm{f}_{\text {energia }}=\Delta \mathrm{t} \cdot \sum_{\mathrm{i}=0}^{\mathrm{N} \operatorname{lnt}}\left(\sum_{\mathrm{j}=0}^{\mathrm{NBom}} \frac{\mathrm{Y} \cdot \mathrm{Q}_{\mathrm{ij}} \cdot \mathrm{H}_{\mathrm{ij}}}{\eta_{\mathrm{ij}}}\right) \cdot C E_{\mathrm{i}} \\
& \mathrm{Y} \text { : peso especifico del agua } \\
& \mathrm{Q}_{\mathrm{ij}} \text { : caudal trasegado en el intervalo i - esimo en la estacion de bombeo j - esima } \\
& \mathrm{H}_{\mathrm{ij}} \text { : altura de impulsión en el intervalo } \mathrm{i} \text { - esimo de la estacion de bombeo j- esima } \\
& \eta_{i j} \text { : rendimiento en el intervalo } \mathrm{i} \text { - esimo de la estacion de bombeo } \mathrm{j} \text { - esima } \\
& C E_{i} \text { : coste de la energía consumida en el intervalo i - esimo } \\
& \Delta \mathrm{t} \text { : duración de cada intervalo temporal }
\end{aligned}
$$

Eq 4. Función validadora del coste energético

El coste de potencia contratada se compone de dos términos. El primero evalúa la potencia requerida en P6 y el segundo en los periodos desde P5 hasta P2. Se considera que no se contrata potencia en periodo P1. Se realiza esta distinción, porque es lógico pensar que se requerirá de diferente potencia puesto que el punto de funcionamiento de la EB será diferente.

$$
\mathrm{f}_{\text {potencia }}=\operatorname{Max}_{\mathrm{t} \subset \mathrm{P} 6}\left(\sum \mathrm{Pot}_{\mathrm{i}}\right) \cdot \mathrm{CPot}_{\mathrm{P} 6}+\underset{\mathrm{t} \not \subset \mathrm{P} 6}{\operatorname{Max}}\left(\sum \mathrm{Pot}_{\mathrm{i}}\right) \cdot \mathrm{CPot}_{\mathrm{P} 2}
$$

Pot $_{i}$ : potencia instantáne a consumida por la bomba $i$ - esima

$\mathrm{CPot}_{\mathrm{P} 6}$ : coste anual unitario de contrataci on en P6

$\mathrm{CPot}_{\mathrm{P} 2}$ : coste anual unitario de contrataci on en P2 (incluyend o P5 - P2)

\section{Eq. 5 Función validadora del coste de potencia}

La calidad del servicio, depende únicamente del margen de presión, que se define como MP=P-P $P_{\text {Requerida. }}$ Se aplica una penalización lineal por tramos de modo que se aplica un mayor coeficiente si el margen de presión sobrepasa el umbral de MP $\mathrm{Leve}$ como se muestra en Eq. 6

$$
\mathrm{f}_{\text {servicio }}=\sum_{\mathrm{i}=1}^{\mathrm{n} \ln \mathrm{n}} \sum_{\mathrm{j}=1}^{\mathrm{nHyd}} \mathrm{p}\left(\mathrm{Hyd}_{\mathrm{ij}}\right) ; \quad \mathrm{p}(\mathrm{Hyd})=\left\{\begin{array}{ccc}
0 & \text { si } & \mathrm{MP}>0 \\
|\mathrm{MP}| \cdot \mathrm{Pen}_{\text {Leve }} & \text { si } & 0>M P>M P_{\text {Leve }} \\
|\mathrm{MP}| \cdot \text { Pen }_{\text {Grave }} & \text { si } & \mathrm{MP}_{\text {Leve }}>M P
\end{array}\right.
$$

Eq. 6 Función validadora de la calidad del servicio

La altura de bombeo en la metodología original se fijó en $44,5 \mathrm{~m}$ y $25 \mathrm{~m}$ respectivamente obtenidos de un análisis preliminar mediante el modelo GESTAR de la red. Sin embargo, durante el mes de julio fue preciso regar a altura constante de $44,5 \mathrm{~m}$ para satisfacer los caudales y presiones requeridas por los hidrantes. 
Por el contrario se observa como es preferible mantener una altura de bombeo similar $(39,5 \mathrm{~m}$ y $38,3 \mathrm{~m})$ produciéndose un coste energético ligeramente superior pero consiguiendo disminuir el coste en potencia contratada además de satisfacer casi en su totalidad los requisitos de presión.

Únicamente en durante el mes de julio, se observa que hay un hidrante que incurre en un déficit de presión de 1 mca durante 2 horas, lo cual es perfectamente asumible.

En las figuras Fig 2. y Fig 3. se muestran de forma consecutiva los resultados obtenidos para cada uno de los días tipo que representan los principales meses de la campaña, desde mayo hasta septiembre. En ellos se muestra la distribución de caudales y el margen de presión del hidrante más desfavorable así como el funcionamiento de la EB que se ha optimizado.

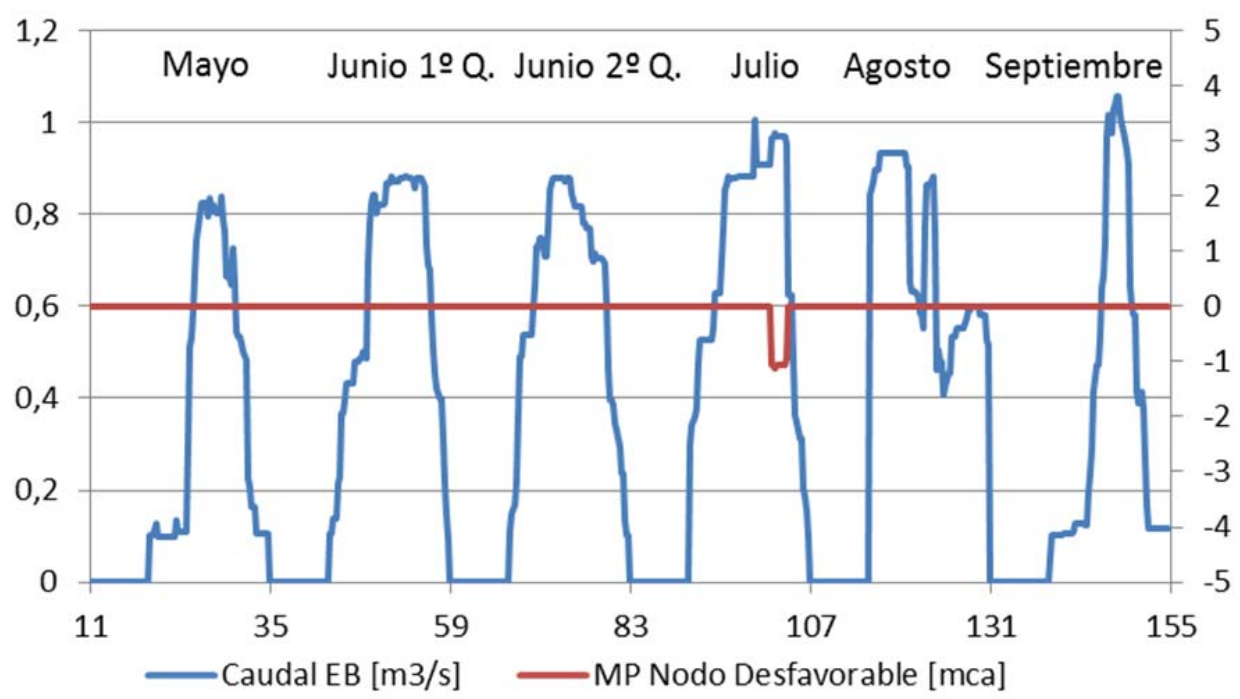

Fig 2. Caudal de la estación de bombeo y margen de presión del Nodo más desfavorable durante cada día tipo de cada mes de la campaña de riego optimizada. 


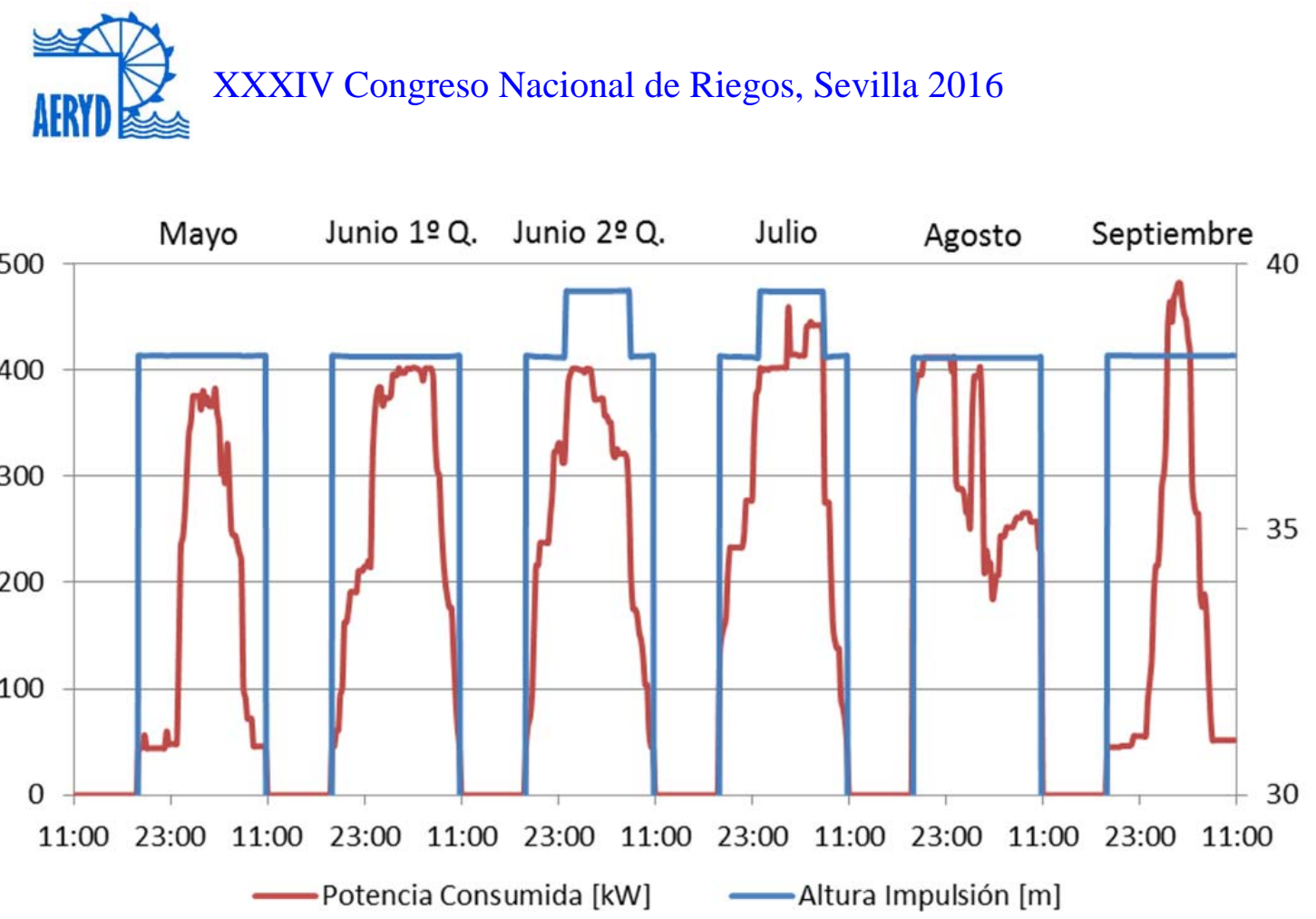

Fig 3. Funcionamiento de la estación de bombeo durante cada día tipo de cada mes de la campaña de riego optimizada.

El tiempo de cálculo, depende en gran medida del tamaño de la red y del número de intervalos en que se haya discretizado. En este caso, la red es pequeña (apenas 50 hidrantes), pero el número de discretizaciones elevadas (casi 600).

El tiempo de cálculo en un ordenador portátil con procesador Intel i7-4710HQ y $16 \mathrm{~Gb}$ de RAM ha sido de apenas 20 minutos. En otros casos, con redes de gran tamaño (en torno a 300 hidrantes) ha supuesto cerca de 2 horas. Por la naturaleza del algoritmo y por cómo se ha implementado, permite su paralelización, por lo que hace uso de todos los cores de la CPU lo que permitiría su utilización en redes de gran tamaño y gran número de discretizaciones.

Por último en la Tabla 1 se muestra una tabla con el resumen de los principales resultados de cada metodología.

Tabla 1. Comparativa de la programación de riego obtenida con ambas metodología.

\begin{tabular}{|l|l|l|}
\hline & Algoritmo original & Algoritmo extendido \\
\hline $\begin{array}{l}\text { Estrategia de } \\
\text { bombeo }\end{array}$ & $44,5 \mathrm{~m} \quad$ P6 +Julio (Impuesta) & $39,5 \mathrm{~m}$ en P6 \\
\hline Potencia contratada & $25 \mathrm{~m} \quad$ Resto P2-P5 (Impuesta) & $38,3 \mathrm{~m}$ en P2-P5 \\
& $\mathrm{P} 2-\mathrm{P} 6 \quad 5 \times 110 \mathrm{~kW}$ (Impuesto) & $\begin{array}{l}\text { P2-P5 4x110kW } \\
\text { P6 5x110kW }\end{array}$ \\
\hline Coste potencia & $38016 €$ & $31132 €(-18 \%)$ \\
\hline Coste energético & $27039 €$ & $27544 €(+2 \%)$ \\
\hline Coste global & $65055 €$ & $58676 €(-10 \%)$ \\
\hline
\end{tabular}

\section{3.- Resultados y Discusión}

El nuevo algoritmo es capaz de optimizar de manera satisfactoria tanto la apertura y cierre como la curva de consigna de la estación conjuntamente. Además de automatizar el proceso, eliminando pasos manuales como la fijación de la potencia 
contratada y de las curvas de consigna que el algoritmo original requería y en un periodo de tiempo aceptable.

\section{4.- Bibliografía}

Aliod R., García S., Faci E., Paño J., Seral P., García A. (2015) "Algorithms and tools for optimum scheduling of on-demand irrigation for an effective energy cost reduction" ICID2015 Conference, Montpellier

Dorigo M. (1992) "Optimization, Learning and Natural Algorithms". Tesis Doctoral, Politecnico di Milano

Estrada, C.; González, C.; Aliod, R.; Paño, J. (2009) “Improved pressurized pipe network for applications in irrigation Systems". Journal of Irrigation and Drainage. ASCE., Vol. 135, No. 4, August 1, 2009..

Faci E. (2014) "Algoritmos evolutivos para la optimización de la gestión hidráulica y energética de redes de riego a presión". Proyecto fin de máster, Universidad de Zaragoza.

García Asin S., Faci E., Aliod R., Paño J., Seral P. (2015) "Algoritmos y herramientas para la aplicación de estrategias de reducción de costes energéticos en sistemas de riego a presión". IV Jornadas de Ingeniería del Agua. Córdoba.

Rossman L. "EPANET 2 USERS MANUAL", Water Supply and Water Resources Division, United States Environment Policy Agency. 